encounter diseases of the oral cavity in their daily practice. The work is current and the references pertinent to the subject matter.

Prof. Ricardo Ambrósio Fock

Faculty of Pharmaceutical Sciences/USP

\section{DRUG ANALYSIS}

BRITTAIN, H.G. Polymorphism in pharmaceutical solids. 2.ed. New York: Informa Healthcare, 2009. 640 p.

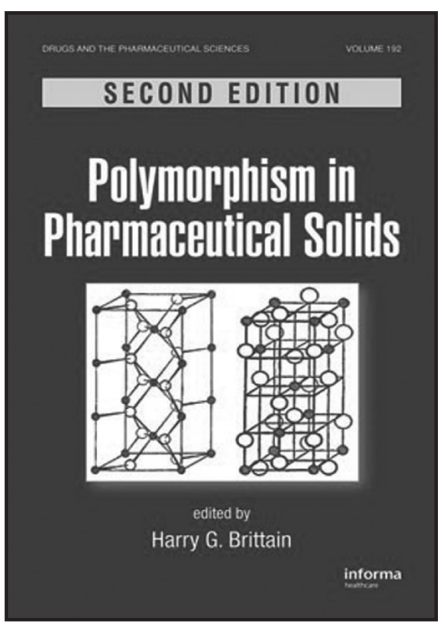

The second edition of "Polymorphism in Pharmaceutical Solids" covers the major topics related to polymorphism in pharmaceuticals. The content of the present edition provides upto-date information on recent developments and methods in characterization and deep studies on the polymorphic state of drug substances. The book is divided into six main sections: the first deals with thermodynamics and theoretical issues including characterization of polymorphic and solvatomorphic systems along with computational methodologies for prediction of the crystal polymorph state of drugs. The second section covers preparative methods for polymorphs and solvatomorphs, where the classical as well as High-Throughput approaches are discussed, with practical examples and the automation potential for scientific and development applications. The structural properties of polymorphs and solvatomorphs, including structural aspects and pharmaceutical co-crystal structures, are discussed in the third section. The characterization of polymorphs and solvatomorphs is a key aspect covered in the fourth section. Modern analytical approaches such as thermoanalytical and crystallographic methods, vibrational spectroscopy, solid-state NMR spectroscopy are included with brief descriptions of the effect of polymorphism and solid-state salvation on important pharmaceutical parameters such as dissolution and solubility of drugs. The fifth section encompasses the interconversion of polymorphs and solvatomorphs as well as the effects of pharmaceutical processing on the solid form of drug and excipient material. The former covers interconversions such as solid-to-solid, dehydration and desolvation, solution and vapor-mediated phase transformation. While the latter embraces processes involved in pharmaceutical manufacture such as milling, granulation, drying, hydration, lyophilization, compression and other shear stress in polymorph and solvatomorph interconversion. Two topics of great interest are covered in the sixth section, namely the structural aspects of molecular dissymmetry, particularly structural variations amongst solids composed of racemates against solids composed of pure enantiomer. Finally, the amorphous solids are discussed in detail, in a separate chapter. This book is ideal for experts from pharmaceutical and chemical research fields as well as for graduate students.

\section{Prof. Dr. Anil Kumar Singh}

Faculty of Pharmaceutical Sciences/USP

\section{FOOD}

CARVIER, B.F. Wheat: Science and Trade. Ames: WileyBlackwell, 2009. 569 p.

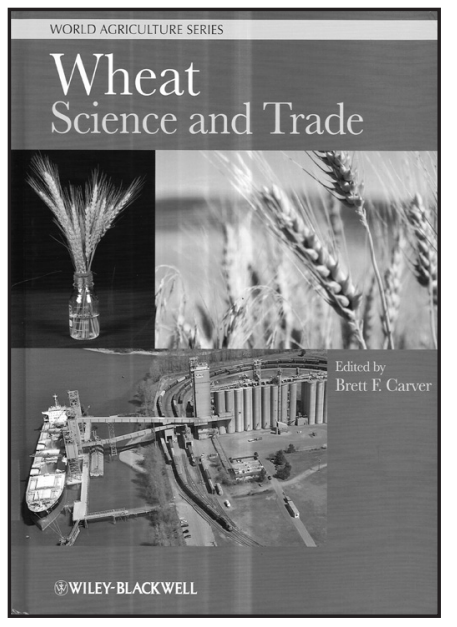

Wheat is the world's largest and most important food crop with an annual harvest of more than 224 million tonnes from 224 million hectares in 2008-2009. It is the greatest source of dietary calories and the most internationally traded agricultural commodity. No other cereal can produce so many different food products as wheat. Over the last 60 years, the increased productivity reached reflects advances in science and technology. These advances are expected to continue in the future, and all over the world there is a challenge to achieve sustainable high-yielding cultures, while avoiding expansion of the area of cultivation. The success of wheat productivitygain programs from favorable irrigated and high-rainfall geographic areas to unfavorable semiarid areas, to meet the demands of an ever-increasing population, depends on all aspects of crop and soil science. Massive investments and sharing of knowledge between private and public sectors of research are the keys for the development of this complex system of wheat production, covering 